\title{
Economía Política de la Nanotecnología
}

\author{
Gian Carlo Delgado Ramos*
}

L

a nanotecnología refiere a la manipulación de la materia a escala na-

nométrica, es decir a la mil millonésima de metro. Se trata de una tecnología que más allá de caracterizarse por operar a esas dimensiones (en la que también trabajan otras disciplinas como la química), particularmente alude al diseño, caracterización y producción de nanoestructuras, nanodispositivos y nanosistemas novedosos a partir de 'controlar' la forma, el tamaño y las propiedades de la materia a dicha escala con el objeto de su uso en diversas aplicaciones civiles y/o militares.

Las aplicaciones nanotecnológicas pueden, por tanto, ser tan distintas y con grados de complejidad tan amplios que los especialistas prefieren hablar de "nanotecnologías" para apreciar con mayor precisión tal diversidad de usos. Por ejemplo, los materiales nanoestructurados ya son utilizados en productos como bolas de tenis, golf o boliche; en la fabricación de neumáticos de alto rendimiento o de telas con propiedades antimanchas / antiarrugas; en cosméticos, fármacos y nuevos tratamientos terapéuticos; en filtros/membranas de agua nanoestructurados y 'remedios' medioambientales; en la mejora de procesos productivos mediante la introducción de materiales más resistentes o eficientes (tanto industriales como agroindustriales); o en el diseño de nuevos materiales para usos que van desde la electrónica, la aeronáutica y prácticamente toda la industria del transporte, hasta para su uso en armas más sofisticadas, ligeras y eficaces.

Estas aplicaciones, entre otras, han generado ya una doble atención. Por un lado, se observan amplios beneficios que posibilitarían la potencial reestructuración, en principio, de todo el entorno material que nos rodea. Y, por el otro, se identifican las posibles implicaciones que esa transformación generaría en el medio ambiente y, de ahí, en la salud, puesto que estarían presentes novedosas nanoestructuras diseñadas por el ser humano y cuyas características, en su gran mayoría, son todavía desconocidas.

Entre los riesgos e implicaciones que ya se indican, inclusive de fuerte tinte éticomoral, están: 1) que la promesa de reducir el consumo de energía y materiales por medio de aplicaciones nanotecnológicas no necesa-

* Investigador del Centro de Investigaciones Interdisciplinarias en Ciencias y Humanidades de la Universidad Nacional Autónoma de México. Contacto: giandelgado@unam.mx 
riamente se realizará si se toma en cuenta toda la 'mochila ecológica' que cuesta la producción, uso y desecho de tales o cuales avances; 2) que las nanoestructuras pueden y seguramente alterarán el organismo humano y el de otras formas de vida con consecuencias impredecibles., por ejemplo, daños a los tejidos, a la información genética de las células, etc; 3) que los potenciales beneficios no necesariamente llegarán a la gran mayoría de la población que carece de medios económicos; 4) que las aplicaciones policiacomilitares potencialmente afectarán los derechos humanos al transformar la naturaleza de la guerra, las operaciones clandestinas, así como también los operativos de contrainsurgencia; o 5) que el actual avance de la nanotecnología, a la par de la biotecnología, la electroinformática y las ciencias cognitivas (las llamadas "tecnologías convergentes") podrían resultar en el mediano-largo plazo en la transformación de la "naturaleza" humana al alterar el cuerpo y la mente, supuestamente hacia algo "mejor" (lo que sea que ello sea), entre otros puntos (véase: Delgado, 2008).

De cualquier modo, las expectativas se mantienen álgidas, factor que se refleja en un gasto público y privado exponencial y en un sostenido impulso y avance de la IyD de las nanotecnologías.

Se estima que las principales aplicaciones que se están desarrollando corresponden al sector salud y las ciencias de la vida en un $18 \%$ del total mundial; al de químicos en un 12\%; al de la industria de la información y las comunicaciones en un 9\%; a de remediaciones y otras aplicaciones medioambientales en un 9\%; al sector transporte en un 8\%; al de energía en un $8 \%$ y al de bienes de consumo en un $8 \%$. Otras áreas de interés son el de la construcción (6\%), artículos del hogar (5\%), defensa y seguridad $(5 \%)$, aeroespacial $(5 \%)$, cuidado personal (3\%), textiles (3\%), y alimentos (1\%) (Nanospots, 2007: 39).

Es de notarse, sin embargo, que a pesar de que ya son numerosos los productos derivados de la nanotecnología que se comercializan o que están en fases de pre-comercialización, éstos son relativamente pocos en comparación a las dimensiones de la inversión efectuada. El grado de retorno de ganancias es todavía mínimo, sobre todo de frente al esperado en el mediano-largo plazo puesto que los diversos datos indican que, en términos del mercado global, hay una tendencia exponencial de negocio: de entrada, en los próximos 10 años, con aplicaciones nanotecnológicas en alrededor del 15\% de la manufacturas a nivel global (Nordan, 2005). A ello súmese las expectativas que se tienen en el ámbito militar, mismo que no necesariamente opera bajo los lineamientos del mercado puesto que, por el contrario, muchas de las veces el producto ya ha sido vendido antes de su desarrollo y producción. Véase cuadro 1 referente a las principales líneas de investigación y desarrollo militar en Estados Unidos. 
CUADRO 1. Gasto en Nanotecnología del Departamento de la Defensa por aplicación (millones de dólares)

\begin{tabular}{|c|c|c|c|c|c|}
\hline Aplicación & 2006 (real) & $\begin{array}{c}\text { Adiciones del } \\
\text { Congreso (2006) }\end{array}$ & $\begin{array}{c}2007 \\
\text { (estimado) }\end{array}$ & $\begin{array}{c}\text { Adiciones del } \\
\text { Congreso } \\
\text { (2007 est) }\end{array}$ & 2008 (solicitado) \\
\hline $\begin{array}{l}\text { Nano-fenómenos y } \\
\text { procesos básicos }\end{array}$ & 184.59 & 33.78 & 180.99 & 33.49 & 179.12 \\
\hline Nanomateriales & 109.70 & 17.46 & 84.77 & 3.06 & 91.68 \\
\hline $\begin{array}{l}\text { Nanodispositivos y } \\
\text { sistemas }\end{array}$ & 110.44 & 21.58 & 107.53 & 23.14 & 70.63 \\
\hline $\begin{array}{l}\text { Instrumental de } \\
\text { Investigación / Metrología }\end{array}$ & 10.77 & 2.73 & 9.51 & 1.37 & 8.25 \\
\hline Nanomanufactura & 3.12 & 0.00 & 4.83 & 1.91 & 1.00 \\
\hline $\begin{array}{l}\text { Instalaciones y adquisición } \\
\text { de instrumentos }\end{array}$ & 4.31 & 0.00 & 28.60 & 0.00 & 22.97 \\
\hline Dimensiones Sociales & 1.00 & 0.00 & 1.00 & 0.00 & 1.00 \\
\hline TOTAL & 423.95 & 75.56 & 417.26 & 62.99 & 374.68 \\
\hline
\end{tabular}

Fuente: Departamento de la Defensa, 2007.

Según Lux Research, en 2004 se registraron 12,980 millones de dólares (mdd) en ventas de productos que utilizan algún tipo de nanotecnología, monto que para 2008, según Cientifica, se especula en 166 mil mdd (Lux Research, 2008; Científica, 2008). Las proyecciones en el corto plazo son aún más llamativas en términos de su potencial ritmo de crecimiento. Para Lux, en 2010 se espera que las ventas asciendan a unos 507 mil mdd (Lawrence, 2005; Baker et al., 2005). Cinco años después, en 2015, se calcula ya el billón de dólares (millón de millones) (Feder, 2004).

En este contexto de entusiasmo nanotecnológico, numerosos esquemas e iniciativas gubernamentales han sido implementados como mecanismos para estimular la investigación y desarrollo de la nanociencia y la nanotecnología (Ver cuadro 2 para el caso de los países a la cabeza de este proceso). En lo que refiere al financiamiento, se estima que el gasto de los gobiernos en nanotecnología a nivel mundial, pasó de 430 millones de dólares en 1997 a 3 mil mdd en 2003; contexto en el que EUA aportó el 25 por ciento (Roco, 2004). Para 2004, Lux Research supone que el gasto total mundial ascendió a 8.6 mil mdd de los cuales 4.6 mil mdd provinieron del sector público. El gobierno de los Estados Unidos de América, EUA, contribuyó con 1.6 mil mdd (1.15 mil mdd a nivel federal y el resto a nivel estatal); el de Japón con alrededor de un millardo; y la Unión Europea, UE, con poco más de mil mdd (350 millones a nivel europeo, y a nivel nacional: 271 millones de Alemania, 187 millones de Francia, 162 millones del Reino Unido y el resto de otros países miembros). 
CUADRO 2. Nanotecnología y su financiamiento: el caso de Estados Unidos, Europa y Japón.

\begin{tabular}{|c|c|c|}
\hline País & Áreas Prioritarias & Política de financiamiento y monto \\
\hline Estados Unidos & $\begin{array}{l}\text { Fenómenos y procesos a la nanoescala; nanomate- } \\
\text { riales; nanodispositivos y nanosistemas de uso civil } \\
\text { y militar; instrumentos de investigación, metrología } \\
\text { y estandarización; nanomanufactura; emplazamien- } \\
\text { to de infraestructura de gran dimensión; grupos de } \\
\text { análisis sobre dimensiones sociales de la nanotec- } \\
\text { nología. }\end{array}$ & $\begin{array}{l}\text { Financiamiento anual desde la Iniciativa } \\
\text { Nacional de Nanotecnología. Presupuesto } \\
\text { estimado para el 2008: } 1.44 \text { mil millones } \\
\text { de dólares. }\end{array}$ \\
\hline $\begin{array}{l}\text { Unión Europea } \\
\text { Comisión Europea -FP7 }\end{array}$ & $\begin{array}{l}\text { Nanociencias y nanotecnología civil y militar; na- } \\
\text { nobiotecnología/nanomedicina (instrumental, } \\
\text { materiales y procesos); nanoelectrónica y optoelec- } \\
\text { trónica; materiales y tecnologías para el desarrollo } \\
\text { de nuevos procesos productivos. }\end{array}$ & $\begin{array}{l}\text { Programa Marco Siete (FP7) del } 2007 \\
\text { al } 2013 \text {. Otorga un financiamiento pro- } \\
\text { gramado de entre } 45 \text { y } 50 \text { mil millones } \\
\text { de dólares. Corresponde sólo al } 31 \% \text { del } \\
\text { gasto total europeo en el rubro. El resto lo } \\
\text { comprende en un } 57 \% \text { el gasto público por } \\
\text { país; en un 6\% programas Comisión Euro- } \\
\text { pea-Estado Nacional; } 4 \% \text { Nacional-Priva- } \\
\text { do; y en } 2 \% \text { Comisión Europea-Privado. } \\
\text { Los países líderes son Alemania (NanoRed } \\
\text { con numerosos entes realizando IyD y más } \\
\text { de medio millar de empresas; cuenta con } \\
\text { la denominada "NanoIniciativa } 2010 \text { "); } \\
\text { Francia (Consorcio en Nanotecnología } \\
\text { encabezado por el Centro Nacional de la } \\
\text { Investigación Científica); y el Reino Unido } \\
\text { (con una Iniciativa Nacional en Nanotec- } \\
\text { nología y su Programa de Nanotecnología } \\
\text { LINK). } \\
\text { El gasto anual alemán programado para el } \\
\text { periodo } 2006-2009 \text { ronda entre los } 300 \text { y } \\
\text { los } 330 \text { millones de euros. El francés los } \\
140-150 \text { millones según estimaciones que } \\
\text { incluyen el } 2008 \text { Y Y el inglés, los } 50 \text { a } 75 \\
\text { millones de euros anuales estimados para } \\
\text { el lapso que va del } 2003 \text { al } 2009 .\end{array}$ \\
\hline Japón & $\begin{array}{l}\text { Nanotecnología relacionada al desarrollo de mate- } \\
\text { riales y dispositivos útiles, sobre todo, a la industria } \\
\text { de la información y las comunicaciones, pero tam- } \\
\text { bién al desarrollo de nuevas energías y tecnologías } \\
\text { ambientales; aplicaciones medicas puntuales; entre } \\
\text { otras áreas. }\end{array}$ & $\begin{array}{l}\text { Tercer Plan Básico de Ciencia y Tecnología } \\
2006 \text { - } 2010 \text { con un financiamiento total } \\
\text { de } 25 \text { billones de yenes o unos } 208 \text { mil } \\
\text { millones de dólares. El gasto en nanotec- } \\
\text { nología programado para el periodo } 2006 \text { - } \\
2010 \text { se estima en unos } 5 \text { mil millones de } \\
\text { dólares. }\end{array}$ \\
\hline
\end{tabular}

Fuente: elaboración Propia en base a datos de: Delgado, 2008 y Nanosposts, 2007. 
En 2005 los montos sugieren haber aumentado a 5.9 mil mdd del sector público, 4.5 mil mdd del gran y mediano empresariado y unos $500 \mathrm{mi}$ llones de start-ups para un total de 10.9 mil mdd (Lux Research, 2006). Para 2006 las cifras de Lux Research precisan un total de 12.4 mil mdd: 6.4 mil mdd del sector público, 5.3 del gran y mediano empresariado y unos 700 millones de start-ups (Holman, 2007). Para el 2007, el monto del gasto total en nanociencia y nanotecnología a nivel mundial se especula entorno a los 14 mil mdd.

Nótese que para propósitos comparativos con otros países como China o Corea del Sur, las cifras deben ajustarse a lo que cada monto compra a nivel local pues, por ejemplo, el gasto gubernamental de China en nanotecnología de 130 mdd para 2004, relativamente compraba lo que en EUA hacían unos 611 millones. $^{2}$

CUADRO 3. Gasto Público en Nanotecnología por País -2004 / millones de euros-

\begin{tabular}{|l|l|}
\hline EUA & 910 (Federal) +333.3 (Estatal) \\
\hline UE & 915 (Estado) +370 (Comisión) \\
\hline Japón & 750 \\
\hline China - Taiwan & $83.3+75.9$ \\
\hline Total & 3,437 \\
\hline Total Mundial & 3,850 \\
\hline
\end{tabular}

Fuente: Comisión Europea, 2005.

En 2006 esa misma relación representó un equivalente a 906 millones (Ibid). Si a lo anterior se suma el gasto de Taiwan de poco más de $100 \mathrm{mi}$ llones de dólares y el de Hong Kong en el rango de otra cifra similar, China y sus provincias "especiales" — con las que tiene acuerdos de cooperación en investigación nanotecnológica- se colocan en términos relativos como "un" competidor importante en la arena internacional aunque en términos absolutos no lo figure así (Cuadro 3). Ello ya es especialmente visible en el rubro de publicaciones en nanociencia y nanotecnología. Véase cuadro 4.

${ }^{2}$ Debe tenerse en cuenta que tal ventaja es sólo en aspectos como salarios y ciertos costos de material de trabajo y de construcción de instalaciones. Algunos componentes y herramientas de trabajo han de ser importadas a precios en dólares o euros, lo mismo sucede con la movilidad internacional de sus investigadores, etcétera. Véase: Nordan, 2005. Op cit. 
CUADRO 4. Posicionamiento en publicaciones de Nanotecnología por autores (1995 - 2005)

\begin{tabular}{|l|r|r|r|r|}
\multicolumn{1}{|c}{ País } & \multicolumn{1}{c}{$\begin{array}{c}\text { Todos los autores } \\
\text { (1995) }\end{array}$} & \multicolumn{1}{c}{$\begin{array}{c}\text { Primer autor } \\
\text { (1995) }\end{array}$} & $\begin{array}{c}\text { Todos los autores } \\
\text { (2005) }\end{array}$ & \multicolumn{1}{c|}{$\begin{array}{c}\text { Primer autor } \\
\text { (2005) }\end{array}$} \\
\hline UE $(27)$ & $3,797(25.3 \%)$ & $3,476(23.2 \%)$ & $17,343(31.0 \%)$ & $14,806(26.4 \%)$ \\
\hline EUA & $3,112(20.8 \%)$ & $2,836(18.9 \%)$ & $14,247(25.4 \%)$ & $12,183(21.8 \%)$ \\
\hline Japón & $1,146(7.6 \%)$ & $1,031(6.9 \%)$ & $6,191(11.1 \%)$ & $5,342(9.5 \%)$ \\
\hline China & $507(3.4 \%)$ & $472(3.1 \%)$ & $9,859(17.6 \%)$ & $9,252(16.5 \%)$ \\
\hline Alemania & $1,077(7.2 \%)$ & $894(6.0 \%)$ & $4,910(8.8 \%)$ & $3,458(6.2 \%)$ \\
\hline Tigres Asiáticos & $351(2.3 \%)$ & $315(2.1 \%)$ & $5,366(9.6 \%)$ & $4,760(8.5 \%)$ \\
\hline
\end{tabular}

Fuente: Youtie ET AL., 2008.

En adición a lo anterior, debe tenerse presente que el gasto en nanotecnología, por un lado, varía según la definición de ésta en cada país (por ejemplo a diferencia de la Unión Europea, en Estados Unidos ninguna actividad de la microelectrónica es sujeta a financiamiento en nanotecnología).

Por el otro lado, hay que también considerar que cuando se habla de gasto en nanotecnología, solamente se trata de aquel cuya modalidad es directa y específica ya que ahí no se considera el gasto indirecto en ciencia y otras tecnologías fundamentales para el avance de la nanotecnología (e.g. física, química, biotecnología). En tal sentido, la fortaleza del aparato científico-tecnológico como un todo es un factor más ha considerar. Y es que si bien los porcentajes de adjudicaciones en la Oficina Europea de Patentes durante 1978-2005 es similar en nanotecnología para EUA (34.6\%), Japón (29.2\%) y Europa (28.4\%) (Igami y Okazaki, 2007: 14), ello no es así en términos generales de la innovación tecnológica de vanguardia ya que EUA acapara el 33\% de ésa a nivel mundial, mientras que la Unión Europea (de los 15 ) lo hace en un $28.8 \%$ y Japón en 12.9 por ciento (NSF, 2004).

En resumen, el estímulo y expectativas en cuanto al avance de la nanociencia y las nanotecnologías es claramente creciente. Involucra actores de múltiples países que se vienen posicionando en diversas áreas o nichos de oportunidad aún a pesar de ya hay actores con clara ventaja. Y es que el potencial de negocio es de gran envergadura.

En el proceso, el diálogo social entorno a los aspectos legales, éticos, sociales y ambientales se considera fundamental puesto que debe incluir a todas las "partes", desde nanotecnólogos y científicos de las ciencias 
'exactas' y naturales, hasta especialistas de las ciencias sociales y las humanidades; empresarios; actores de la esfera política nacional e internacional; representantes de la sociedad civil; etcétera (Delgado, 2008: 381-417). El objeto: maximizar beneficios considerando las realidades nacionales; evitar o minimizar costos innecesarios; y consensuar y distribuir el riesgo.

\section{BibLIOGRAFIA}

Baker, Stephen y Aston, Adam (2005). "The Business of Nanotech." Business Week. EUA, 14 de enero.

Comisión Europea (2005). Some Figures about Nanotechnology R\&D in Europe and Beyond. Bruselas, Bélgica.

Delgado, Gian Carlo (2008). Guerra por lo Invisible: negocio, implicaciones y riesgos de la nanotecnología. Ceiich, UNAM. México, 2008.

Departamento de la Defensa (2007). Defense Nanotechnology Research and Development Program. EUA, 26 de abril.

Feder, Barnaby (2004). "Nanotech IPOs, without a product." The Internacional Herald Tribune. 26 de mayo.

Holman, Michael (2007). Top Nations in Nanotech See Their Lead Erode. Lux Research. EUA, 8 de marzo.

Igami, Masatsura y Okazaki, Teruo (2007). Capturing Nanotechnology's Current State of Development Via Analysis of Patentes. STI Working paper 2007/4. Directorate for Science, Technology and Industry. OECD. 23 de mayo.

Lawrence, Stacy (2005). "Nanotech Grows Up. Funding for R\&D doubled in 2004.” Technology Review. EUA.

Lux Research (2006). The Nanotech Report. Investment Overview and Market Research for Nanotechnology. Lux Research. Nueva York, EUA.

Científica (2008). Nanotechnology Opportunity Report. 3rd edition. EUA.

Nanoposts (2007). Government Policy and Initiatives in Nanotechnology Worldwide 2007. Canadá.

Nordan, Matthew (2005). "Nanotechnology: where does the US stand?" Lux Research. Testimonio ante la House Committee on Sciences. EUA, 29 de Junio.

NSF - National Science Foundation (2004). Science \& Engineering Indicators 2004. Vol. 2. EUA 
Roco, Mihail (2004). "Nanoscale science and engineering: unifying and transforming tools." AIChE Journal, Vol. 50. No. 5. EUA.

Youtie, Jan; Aspira, Philip; Porter, Alan L (2008). "Nanotechnology publications and citations by leading countries and blocs". Journal of Nanoparticle Research. SpringerLink. Holanda. 